\title{
Overloaded and fractured stellar winds
}

\author{
Achim Feldmeier, Lida M. Oskinova, Wolf-Rainer Hamann \\ Lehrstuhl Astrophysik, Institut für Physik, Universität Potsdam, \\ Am Neuen Palais 10, D-14469 Potsdam, BRD
}

Stanley P. Owocki

Bartol Research Institute, University of Delaware, 217 Sharp Laboratory, Newark, DE 19716, USA

\begin{abstract}
We discuss the connection between wind overloading and discrete absorption components in P-Cygni line profiles from O-type stars. Overloading can create horizontal plateaus in the radial wind speed that cause the extra absorption in the line profile. The upstream propagation speed of these velocity plateaus is analyzed. The second part of the paper deals with X-ray emission from O-type stars. X-ray line profiles observed with Chandra and XMM-Newton are often symmetric, contrary to what is expected for lines from a homogeneous wind. We discuss the influence on line symmetry of photon escape channels in a strongly clumped wind.
\end{abstract}

\section{Overloaded winds}

Many OB stars show propagating, discrete absorption components, DACs, in their UV P-Cygni line profiles (Kaper et al. 1996 and references therein). These DACs are probably caused by corotating interaction regions (CIRs) in the wind (Mullan 1984). Numerical O-type star wind simulations show that not the CIR density enhancement itself causes a DAC, but an accompanying velocity plateau that lies starwards (Cranmer \& Owocki 1996). Note that the Sobolev line optical depth scales as $\propto \rho /(d v / d r)$, and can be large either due to large gas densities or shallow velocity gradients. The velocity plateau propagates upstream in a fast, supercritical flow, hence is advected outward at a speed slower than the wind speed.

A remarkable property of observed DACs is that both their propagation and recurrence time (of order one day) are longer than the wind flow time (few hours). While certainly longer than the wind flow time, the timescale of CIR-induced DACs is not fully understood. On its starward facing side, the velocity plateau causing the DAC joins the inner, steeply accelerating wind via a weak discontinuity, i.e., a jump in $d v / d r$ or a kink. In gas dynamics, weak discontinuities propagate at the characteristic wave speed, whereas strong discontinuities in $v$ and $\rho$ itself or shocks propagate (much) faster. Since the radiative line force in O-type star winds depends nonlinearly on $d v / d r$, it appears that not $v$ itself is the fundamental fluid variable in a characteristic analysis, but instead $d v / d r$ (Abbott 1980; Bjorkman 1995). 
Our question is therefore: do kinks in line driven flows propagate upstream as weak discontinuities at the radiative-acoustic wave speed, or as strong discontinuities and therefore much faster? To answer this question, we study kink propagation in 1-D flow simulations. To form kinks in a 1-D flow without CIRs, we artificially create a second critical point in the flow, above the original CAK critical point (Castor et al. 1975).

For the Laval nozzle, critical points coincide with minima of the nozzle area or throat function. This can be generalized to stellar winds, and the generalized throat function is then proportional to the flow tube area, the radiative flux, and inverse gravity (Abbott 1980). A wind that is launched subsonic in the photosphere reaches critical wave speed at the first nozzle minimum. What happens at the second minimum? As suggested by Holzer (1977) for the Laval nozzle or the solar wind, a deep minimum, i.e., a narrow throat, causes the flow to become mass overloaded, and a shock forms.

Line driven winds, by contrast, need not to develop a shock (strong discontinuity), but can overcome the second, overloaded constriction by switching to a decelerating solution, $d v / d r<0$. In Sobolev approximation, this switch occurs via a weak discontinuity, i.e., a kink, in the velocity law. The left panel of Figure 1 shows an O-type star wind forming a steady kink shortly before the second throat minimum.

What happens if the throat is made ever narrower? Eventually, the flow decelerates so strongly that negative speeds occur. Gas falls back to the photosphere and collides with gas streaming upward, resulting in gas compression and shocks. This bears resemblance to the model by Howk et al. (2000), who suggest that backfalling blobs occur in the wind of $\tau$ Sco, causing the hard Xray emission from this star. But whereas Howk et al. suggest that backfalling clouds surround the star at all times in a kind of quasi-steady equilibrium, we find that the flow avoids mass overloading. A shock forms at the second throat minimum and propagates inward. Interestingly, this shock can pass through the inner, CAK critical point, the first throat minimum. The shock reaches the photosphere and adapts it to the mass loss rate sustainable at the outer nozzle minimum. The resulting flow, shown in the right panel of Figure 1, is a breeze at the inner throat minimum (the former CAK critical point), and is a critical transition for radiative-acoustic waves at the outer minimum. No overloading occurs.

In the near future, we shall analyse kink propagation in more detail using this 1-D flow model, in order to better understand the timescale of observed DACs in UV P-Cygni line profiles.

\section{Fractured winds}

There is, on large, twofold evidence for clumping and shock production in hot star winds: blob turbulence (Moffat 1994) and X-ray emission (Lucy \& White 1980). Wind shock models are challenged by Chandra and XMM-Newton observations of X-ray emission lines. If these lines indeed originate in the wind, photons from the stellar back hemisphere (with respect to the observer) have a longer pathway through the wind than front hemisphere photons. The red line wing should then be stronger suppressed than the blue wing. In contrast, many of the observed X-ray line profiles are symmetric. 

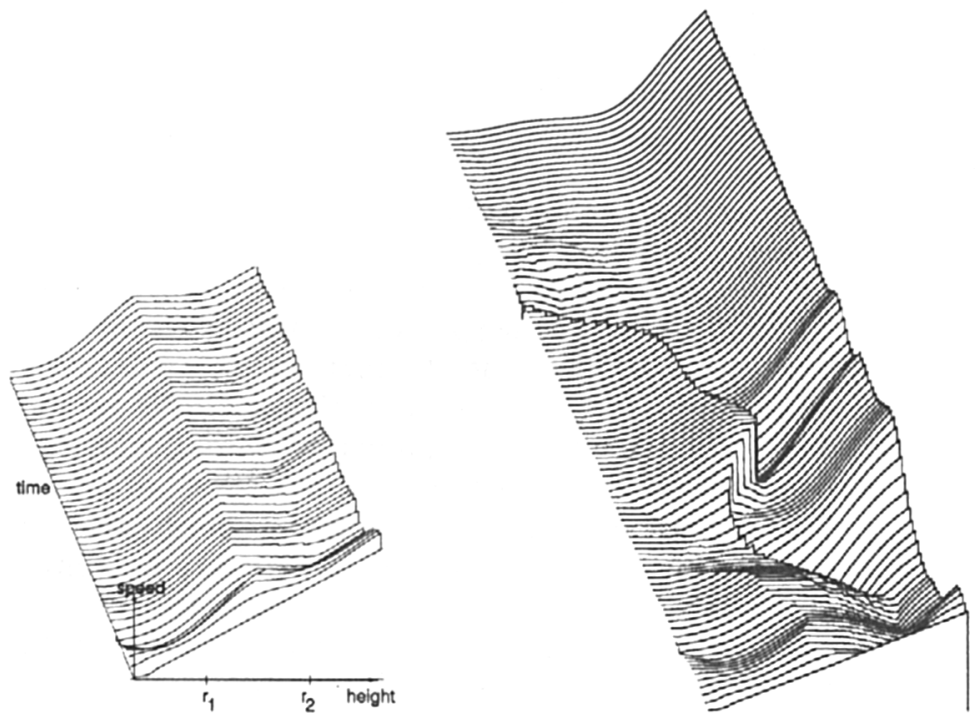

Figure 1. Left: temporal evolution of the flow speed $v$ of a line driven wind. The flow passes through two minima in the nozzle function, at $r_{1}$ and $r_{2}$. The first minimum is a critical transition. (CAK critical point), the second one is mass overloaded. The wind switches to a decelerating solution before the second nozzle minimum, forming a steady kink in $v(r)$. Right: the same flow, with a deeper second nozzle minimum. Flow deceleration results in negative speeds. A shock propagates to the photosphere and adapts it to the outer nozzle minimum, which becomes now critical. No overloading remains.

Two effects could help to make X-ray lines appear symmetric: first, Xrays probably originate in reverse shocks on the starward facing side of dense shell fragments. Therefore, photons from the near hemisphere can be absorbed by their natal shell, whereas back hemisphere photons cannot. Second, if the wind gas is concentrated in a small number of highly compressed shells, back hemisphere photons can pass freely between two shells at low altitude.

We set up a simple model of a fractured, X-ray emitting wind, shown in Figure 2. A finite wind sphere of normalized radius 1 is cut into $N_{\phi}$ wedges of constant opening angle $\delta \phi=2 \pi / N_{\phi}$. Each wedge of angular extent $\pi$ is cut into $N_{\theta}=N_{\phi} / 2$ cones of opening angle $\delta \theta=\delta \phi$. In each cone reside $N_{r}$ spherical shell fragments, distributed randomly and uniformly between radii 0 and 1 . They could result from fragmentation of highly compressed shells formed by the line deshadowing instability. We assume that the fragments are 2-D layers of infinite compression. The photons propagate along the wedges, the observer is located at $\theta=0$. The number of wedges and cones shall be large, but $N_{r}$ may be small. 


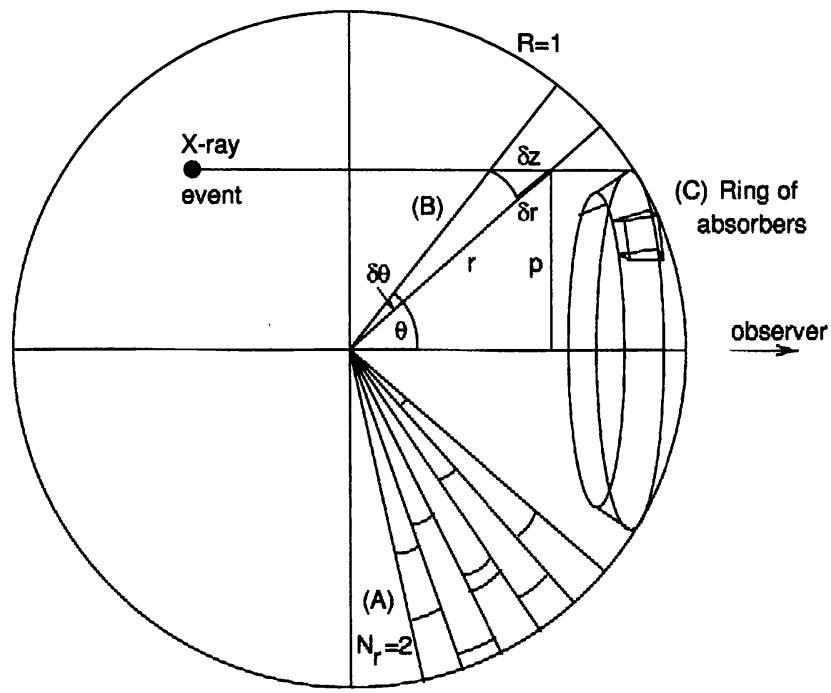

Figure 2. Fractured wind geometry. (A) A finite wind sphere is cut into cones of opening angle $\delta \theta=\delta \phi$, with $N_{r} \geq 1$ geometrically thin shell. fragments in each cone. (B) The hit probability $\delta w$ for a single fragment equals the normalized distance $\delta r$ the photon travels through a cone. (C) For each impact parameter $p$ and latitude $\theta$, the shell hits along an azimuthal ring of cubes with edges $[r \delta \theta, r \sin \theta \delta \phi, \delta r]$ are Poisson distributed.

We assume for the moment $N_{r}=1$ randomly distributed shell fragments per cone. The probability, $\delta w$, that the photon hits such a fragment is given by the radial distance the photon travels through the cone, divided by the cone length. From Figure 2,

$$
\delta w=p \frac{\cos \theta \delta \theta}{\sin ^{2} \theta},
$$

with normalized impact parameter $p$ of the photon ray. The hit probability $\delta w$ goes to zero with the opening angle $\delta \theta$ of the cone. At the same time, the number of azimuthal cones with given $p$ and $\theta$ goes to infinity. These are the requirements for a Poisson distribution: small event probability per trial plus large number of trials. Note that the wedges along which the photons propagate are mutually independent. The single parameter of the Poisson distribution is its expectation value, $\lambda$,

$$
\lambda=N_{\phi} \delta w=2 \pi p \frac{\cos \theta}{\sin ^{2} \theta} .
$$

To give an example, for $N_{r}=1, p=1 / 2$ and $\theta=45^{\circ}$, on average $\lambda=\pi \sqrt{2}=4.44$ shell fragments are hit on the ring, independent of $N_{\phi}$. If the number $N_{\phi}$ of azimuthal cones is doubled, $\delta \phi, \delta \theta$, and therefore $\delta w$ drop by a factor of two. The probability $w_{k}$ that $k$ shell fragments are hit along the ring is

$$
w_{k}=\frac{\lambda^{k}}{k !} e^{-\lambda}, \quad k=0,1,2, \ldots
$$

We can now calculate the photon throughput of the ring. Due to different photon histories, the input intensity of each cone differs. For simplicity, we consider here only an average input intensity, $\left\langle I^{i}\right\rangle$. The output intensity, $I^{o}$, is

$$
I^{o}=I_{1}^{o}+\ldots+I_{N_{\phi}}^{o}=N_{\phi}\left\langle I^{o}\right\rangle=\left\langle I^{i}\right\rangle \sum_{k=0}^{\infty} w_{k}\left(N_{\phi}-k+k e^{-\tau}\right),
$$


where $\tau$ is the optical depth of the fragments, assumed to be a function of $r$ only. Using (3) and the binomial formula in (4), and assuming that the number of shell hits is much smaller than the number of cones along the ring, i.e., that the sum converges at small $k$, one finds

$$
\left\langle I^{o}\right\rangle=\left\langle I^{i}\right\rangle e^{-\lambda} \sum_{k=0}^{\infty} \frac{\lambda^{k}}{k !}\left(1-\frac{1-e^{-\tau}}{N_{\phi}}\right)^{k},
$$

This is the power series expansion of the exponential,

$$
\left\langle I^{o}\right\rangle=\left\langle I^{i}\right\rangle e^{-\lambda} e^{\lambda\left(1-\frac{1-e^{-\tau}}{N_{\phi}}\right)}=\left\langle I^{i}\right\rangle e^{-\lambda \frac{1-e^{-\tau}}{N_{\phi}}}=\left\langle I^{i}\right\rangle e^{-\delta w\left(1-e^{-\tau}\right)} .
$$

Using (6), one can calculate optical depths over finite distances in the wind (and for arbitrary $N_{r}$ ), and can perform line synthesis calculations. Details on this will be given in a forthcoming paper. We here only summarize two central results/problems that emerged from these calculations: $(i)$ wind fragmentation can make the line profile appear less asymmetric. But the basic asymmetry remains, the blue emission line wing is always stronger than the red wing; and (ii) if absorption in the natal shell is included, the line profile becomes black around rest-frame frequency. Effectively double-peaked line profiles result, which are however not observed. This is further discussed in the paper by Oskinova et $a l$. in these Proceedings.

Acknowledgments. Work in this project was funded by the Deutsche Forschungsgemeinschaft under grant Fe 573/1-1. We thank Isaac Shlosman, Joachim Puls and Robert Nikutta for illuminating discussions.

\section{References}

Abbott, D.C. 1980, ApJ 242, 1183

Bjorkman, J.E. 1995, ApJ 453, 369

Castor, J.I., Abbott, D.C., Klein, R.I. 1975, ApJ 195, 157

Cranmer, S.R. Owocki, S.P. 1996, ApJ 462, 469

Holzer, T.E. 1977, J. Geophys. Research 82, 23

Howk, J.C., Cassinelli, J.P., Bjorkman, J.E., Lamers, H. 2000, ApJ 534, 348

Kaper, L., Henrichs, H.F., Nichols, J.S., et al. 1996, A\&AS 116, 257

Lucy, L.B., White, R.L. 1980, ApJ 241, 300

Moffat, A.F. 1994, Reviews in Modern Astron. 7, 51

Mullan, D.J. 1984, ApJ 283, 303 